\title{
Mechanisms linking childhood weight status to metabolic risk in adolescence
}

\author{
Suzanna M. Martinez ${ }^{1,2}$ @ | Estela Blanco ${ }^{2,3}$ | Raquel Burrows ${ }^{4}$ | Betsy Lozoff ${ }^{5}$ | \\ Sheila Gahagan ${ }^{2}$
}

${ }^{1}$ Division of Epidemiology and Biostatistics, University of California San Francisco, San Francisco, CA

${ }^{2}$ Department of Pediatrics, Division of Child Development and Community Health, University of California San Diego, La Jolla, CA ${ }^{3}$ Public Health PhD Program, University of Chile, Santiago, Chile

${ }^{4}$ Institute of Nutrition and Food Technology, University of Chile, Santiago, Chile

${ }^{5}$ Department of Pediatrics and Communicable Diseases, University of Michigan, Ann Arbor, MI

Correspondence

Suzanna M. Martinez, 550 16th Street, Mission Hall, 2nd Floor, San Francisco, CA 94143.

Email: suzanna.martinez@ucsf.edu

Funding information

National Heart, Lung, and Blood Institute, Grant/Award Numbers: K01HL129087, R01HL088530; National Institute of Child Health and Human Development, Grant/ Award Number: R01HD33487

Peer Review

The peer review history for this article is available at https://publons.com/publon/10. 1111/pedi.12972.

\begin{abstract}
Background: Obesity is a risk factor for insulin resistance (IR) and metabolic disease. Objective: To examine potential metabolic pathways linking childhood weight status to adolescent IR and metabolic risk.

Methods: Participants were 600 low- to middle-income Chilean adolescents from a cohort studied since infancy as part of an iron deficiency anemia preventive trial and follow-up study. We examined body mass index z-score at 10 y (BMlz-10y) and blood pressure, total fat, and fasting glucose, adiponectin to leptin ratio (A:L), ghrelin, and HOMA-IR at $16 \mathrm{y}$. A total count for metabolic risk factors (MRF) was calculated using the International Diabetes Federation criteria. We used path analysis to estimate pathways and model indirect effects from BMlz-10y, controlling for child age and sex and maternal body mass index (BMI).

Results: Participants were $54 \%$ male; mean BMlz-10y of 0.53 (SD = 1.02); mean MRF of 1.3 (SD = 0.9); mean HOMA-IR of 1.8 (SD = 1.3). Path analysis showed that BMlz-10y directly and indirectly related to increased MRF via A:L and HOMA-IR. Ghrelin was not in the metabolic pathway from BMlz-10y to MRF but was related to MRF via HOMA-IR.

Conclusion: These results elucidate metabolic pathways involving child weight status, IR and metabolic risk in adolescents. Childhood BMI was an indirect risk factor for adolescent cardiometabolic risk via several pathways that involved BMI, appetite hormones, markers of inflammation, and insulin resistance during adolescence. Findings illustrate the adverse effect that childhood obesity has on adolescent health outcomes, which sets precedence for health outcomes over the life course.
\end{abstract}

\section{KEYWORDS}

adiponectin, adolescents, ghrelin, hormones, insulin resistance, leptin, metabolic risk, weight status

\section{1 | INTRODUCTION}

Childhood is a critical period that can influence health throughout the life course. The impact of childhood weight status on metabolic health extends into adulthood and can last a lifetime. This is in line with the developmental origins of health and disease hypothesis. ${ }^{1}$ There is mounting evidence to suggest that obesity impacts metabolic risk through insulin resistance (IR), ${ }^{2}$ yet the mechanisms are unclear. 
A better understanding of the metabolic mechanisms that connect childhood weight status to later metabolic health is needed to improve clinical strategies that can reduce or prevent health complications throughout the life course.

Studies of adults have established pathways that connect obesity to energy intake and appetite hormone regulation such as ghrelin and leptin. The function of ghrelin is closely related to feeding behavior and the metabolic function of adipokines (such as leptin and adiponectin). Ghrelin functions as an appetite-stimulatory signal, with pre-meal/preprandial increases and postprandial decreases. ${ }^{3}$ Levels of ghrelin show diurnal variations and decrease with food intake, increasing age, body mass index (BMI), glucose and insulin, and are higher in females than males. ${ }^{3}$ Ghrelin also has anti-inflammatory effects. ${ }^{3,4}$ Conversely, leptin functions as a feedback mechanism that rises postmeal (postprandial) and signals the brain to inhibit food intake to regulate body weight and energy balance/homeostasis. Levels of leptin have been found to be higher in individuals with increased weight status and body fat. ${ }^{5}$ Together leptin and ghrelin exert opposing effects on appetite, playing a central role in energy balance.

Insulin and adiponectin are interrelated and both play a protective role in the development of obesity. ${ }^{6}$ Insulin is released into the bloodstream shortly after food intake to lower blood glucose and inhibits appetite similarly to leptin. Adiponectin is secreted by the adipose tissue. ${ }^{7}$ Plasma adiponectins are correlated negatively with insulin resistance. ${ }^{7}$ Moreover, researchers have found low adiponectin levels among individuals with type 2 diabetes, even after adjusting for body weight. $^{8}$

Ghrelin and adipokines (leptin and adiponectin) are related to cardiovascular effects in adults. Leptin is known for its pro-inflammatory function, adiponectin for its anti-inflammatory function, and ghrelin for its role on endocrine pancreatic function, glucose metabolism, and positive vasodilatory effects. ${ }^{8}$ Most studies have examined these factors in isolation, with few studies examining the interrelationships between these factors, and in relation to metabolic risk. ${ }^{8}$ Schutte and colleagues examined the relation of adipokines to metabolic risk ( $N=115$ women ages 29-42) and found a positive relationship between leptin with insulin resistance and a negative relationship with ghrelin and insulin resistance. ${ }^{8}$ Others have found that leptin is positively correlated with BMI but that adiponectin is correlated with decreased BMI in children. ${ }^{9}$ More recently, the ratio of leptin to adiponectin (L:A) was used as a sensitive marker for detecting early metabolic disturbance and insulin resistance. ${ }^{10}$

From previous studies of adults, Lucasson et al. developed a framework to examine how adult lifestyle behaviors, especially sleep loss, lead to the development of chronic disease via endocrine mechanisms that stimulate appetite, decrease energy expenditure, and increase insulin resistance. ${ }^{7}$ In the current study, we applied Lucasson's framework to elucidate mechanisms connecting childhood weight status to metabolic risk in adolescence (Figure 1).

The risk for metabolic syndrome is increasingly a problem among children and adolescents. Therefore, it is essential to understand the mechanisms by which childhood weight status can impact adolescent health. This study aimed to explore potential pathways linking

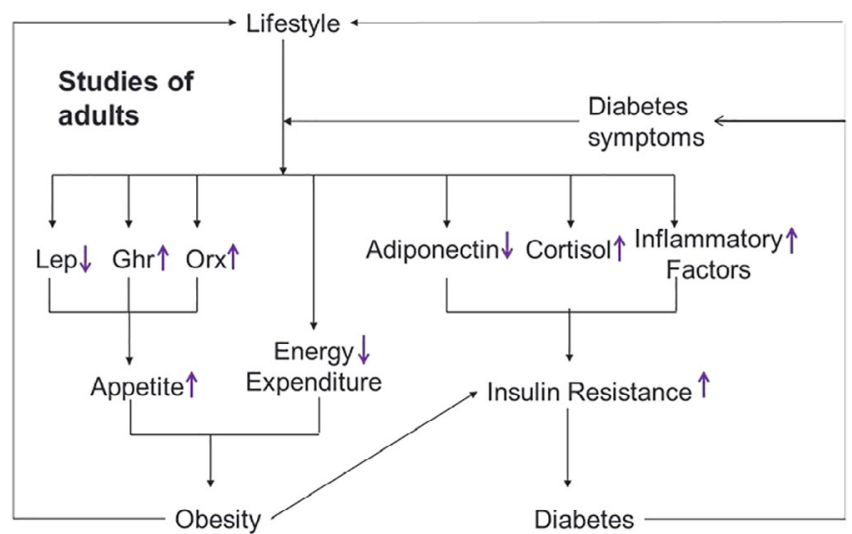

FIGURE 1 An adapted and simplified schematic representation of Lucasson's putative pathways linking lifestyle to obesity and diabetes, via endocrine mechanisms that stimulate appetite, decrease energy expenditure, and increase insulin resistance. The direction of change (an increase or decrease) of each mechanism due to poor lifestyle, such as sleep loss; decreased leptin (lep) and increased ghrelin (ghr) could stimulate appetite; increased appetite could increase obesity risk; decreased adiponectin could increase insulin resistance, and in turn increase diabetes risk

childhood weight status at $10 \mathrm{y}$ to metabolic risk in a cohort of 679 Chilean adolescents. We hypothesized that childhood weight status would be related to an increase in the number of metabolic risk factors through biomarkers of appetite hormone regulation (ghrelin, leptin, and adiponectin) and insulin resistance. We also hypothesized that insulin resistance would be partially mediate the relationship between ghrelin, L:A, and metabolic risk.

\section{2 | METHODS}

\subsection{1 | Study cohort and setting}

Participants were of low- to middle-income backgrounds in Santiago, Chile. Adolescents and their mothers (or caregivers) were participants in an ongoing longitudinal cohort study, which began as an iron anemia deficiency (IDA) preventive trial between 1991 and 1996. ${ }^{11}$ Healthy infants (singleton, full-term births, born via routine vaginal delivery, had birth weight $\geq 3.0 \mathrm{~kg}$, who had no major complications, no chronic illness, and required no iron therapy) were recruited from four contiguous low- to middle-income, working-class, communities located in the southern part of Santiago $(n=1657)$. These neighborhoods were selected based on their close proximity to the research site, the Institute for Nutrition and Food Technology (INTA), University of Chile. Details about the enrollment and trial have been previously described. ${ }^{12}$ The cohort was evaluated at 5,10 , and $16 \mathrm{y}$. At $5 \mathrm{y}$, the available budget allowed evaluation of two of the three infancy randomization groups. We assessed those who had been assigned to iron-supplementation or no iron added $(n=888)$. Thus, those seen at the $5 \mathrm{y}$ follow-up are the most studied group and were invited to participate in an adolescent study of risk for obesity and cardiovascular 
risk factors between 2005 and $2012(n=679){ }^{12}$ Participants in the adolescent follow-up of cardiovascular risk were similar to participants of the original infancy study with respect to the following variables: birth weight and length, sex, maternal and paternal total years of education, maternal age, number of stressors reported in infancy, and maternal CES-D in infancy (all $P>$.05). The adolescent group was breastfed exclusively for slightly longer (118 vs $100 \mathrm{~d}$ ) and ended any breastfeeding slightly later than those in the entire infancy cohort (244 vs $227 \mathrm{~d}$ ), both $P<.05$.

\subsection{2 | Study design}

For this study, we used data collected at two time points between 2005 and 2012 ( $n=679$ ). This study was approved by the Institutional Review Boards at INTA, the University of Michigan, and the University of California, San Diego.

\subsection{3 | Assessment of weight status}

At $10 \mathrm{y}$, height $(\mathrm{cm})$ and weight $(\mathrm{kg})$ were measured by a trained nurse at INTA. Standardized procedures (27) were used to measure weight to the closest $0.1 \mathrm{~kg}$ and height to the closest $0.1 \mathrm{~cm}$. Measurements were taken twice, with a third measurement if the difference between the first two exceeded $0.3 \mathrm{~kg}$ for weight and $0.5 \mathrm{~cm}$ for height. Maternal height and weight were also measured during the child's visit.

Anthropometry was measured in adolescence (between 16 and 18 y) by two trained physicians. Weight and height were assessed in duplicate with a Precision Hispana scale and a stadiometer accurate to $0.1 \mathrm{~kg}$ and $0.1 \mathrm{~cm}$, respectively. Participants were measured without shoes, wearing underwear, in the Frankfurt position. Waist circumference and blood pressure were also measured twice. If the first and second measurements did not match for height, weight, or blood pressure (BP), the measurements were repeated. All participants were measure for $\mathrm{BP}$ according to standard protocols.

BMI z-scores at $10 \mathrm{y}$ and in adolescence were calculated using CDC BMI percentiles, which consider age and sex, and analyzed as continuous variables.

\subsection{4 | Blood assays}

After an overnight fast, a morning blood sample (between 800 and 1000) was collected for the measurement of fasting glucose, lipids, a measure of insulin resistance and appetite hormones. Fasting serum leptin concentration ( $1 \mathrm{ng} / \mathrm{mL}$ sensitivity) was measured using the Sandwich Enzyme Immunoassay kit (DRG International, Inc., USA) and adiponectin using ELISA R\&D Systems (Minneapolis, MN, USA). Leptin to adiponectin ratios were then calculated. Commercial radioimmunoassay kits were used to measure ghrelin (Phoenix Pharmaceuticals, Inc., Burlingame, CA, USA) and insulin (Diagnostic Products Corporation, Los Angeles, CA, USA). Glucose was measured using the enzymatic-calorimetric kit (QCA S.A. Amposta, Spain). HOMA-IR was then calculated as [glucose*insulin]/405. Concentration of high density lipoprotein and triglyceride levels were measured using the analytical methodology dry kit (Vitros, Johnson \& Johnson, Clinical Diagnostics, Inc.). Glucose was measured using the enzymaticcalorimetric kit (QCA S.A. Amposta, Spain).

\subsection{Study variables}

\subsection{1 | Independent variable}

Childhood weight status. We examined BMI z-scores at 10 y (BMIz$10 y)$ as the independent risk factor for metabolic risk.

\subsection{2 | Dependent variable}

Metabolic risk factors. We examined the number of metabolic risk factors for the metabolic syndrome as the dependent variable. We used the IDF criteria to compute the number of MR factors using the following five risk factors: elevated BP, a low high density lipoprotein cholesterol level, a high triglyceride level, a high fasting glucose level, and abdominal obesity, adjusted for age and sex. ${ }^{13}$ BP > 90th percentile was defined as a systolic or diastolic BP > 90th percentile for age, sex, and height.

\subsection{3 | Mediators}

Appetite regulation hormones and inflammation. We examined biomarkers of appetite regulation and inflammation at $16 \mathrm{y}$ as mediators of the relationship between BMlz-10y and metabolic risk factors. This included ghrelin [pg/mL], L:A ratio $[\mu \mathrm{g} / \mathrm{mL}]$, and HOMA-IR.

\subsection{4 | Covariates}

We controlled for child age and sex and maternal BMI at the $10 y$ wave.

\subsection{5 | Analytic framework}

We tested pathways between BMlz-10y to metabolic risk, which was informed by the framework best described by Lucasson et al. (Figure 1) and illustrated in Figure 2.

\subsection{6 | Analytic plan}

Sample characteristics, including BMI and HOMA-IR, were examined for the total sample and by sex. Appetite hormones and energy 


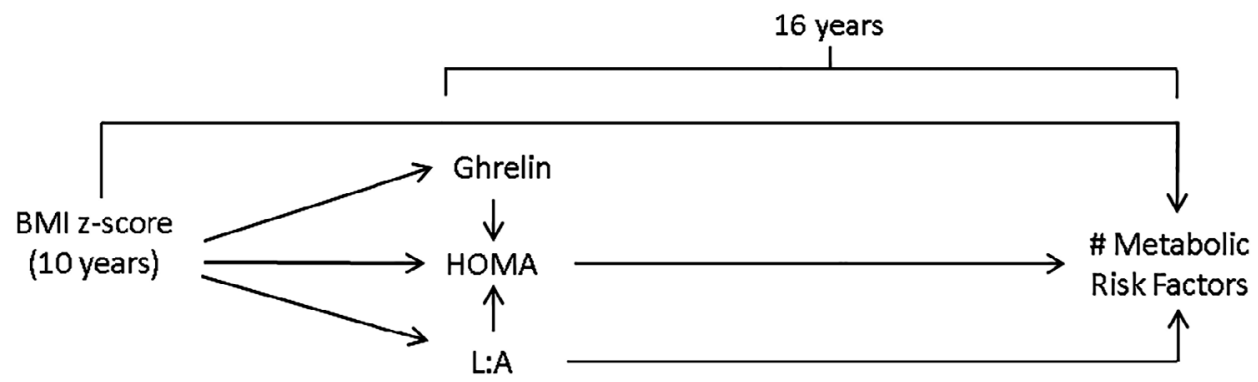

FIGURE 2 Illustration of the potential pathways linking child weight status at age 10 to risk for metabolic syndrome at age 16 regulators were examined by sex and using the 95th percentile cutoff for obesity. Chi-square tests were considered significant at $P<.05$. All descriptive statistics were performed using IBMI SPSS 21.

The main analytic plan involved longitudinal analysis using data from the $10 y$ and $16 y$ waves. We used path analysis to explore pathways between BMIz-10y and metabolic risk factors. The model tested three metabolic pathways: 1) BMlz-10y $\rightarrow$ ghrelin $\rightarrow$ number of metabolic risk factors; 2) BMIz-10y $\rightarrow$ L:A $\rightarrow$ HOMA-IR $\rightarrow$ number of metabolic risk factors; and 3) BMlz-10y $\rightarrow \mathrm{L}: \mathrm{A} \rightarrow$ number of metabolic risk factors. The model also tested the direct effect of BMlz-10y on metabolic risk factors at $16 \mathrm{y}$. First, we conducted a multiple group analysis to account for sex differences, given the empirical evidence that leptin and ghrelin levels differ by sex. Second, because models were similar for females and males, we conducted a one-sample path analysis, which controlled for the relationships of sex and age with ghrelin, L:A, HOMA-IR and metabolic risk, and maternal BMI with BMlz-10y. For parsimony, the final model controlled for covariates with $P<.20$, which included relationships of sex with ghrelin, $L: A$, HOMA-IR and metabolic risk, age with ghrelin and HOMA-IR, and maternal BMI with BMIz-10y. All path analyses was performed using MPlus 7 software (Muthén \& Muthén, Los Angeles, CA), with the full maximum likelihood function. The model fit was determined by using the following indices: Confirmatory fit index (CFI $\leq 0.90$ ); root mean square error of approximation (RMSEA $\leq 0.06$ ); and standardized root mean square residual (SRMR $\leq 0.08$ ); and a significance level of $P<.05$. Mediation was tested using the INDIRECT command to estimate indirect effects, using MPlus.

\section{3 | RESULTS}

\subsection{1 | Sample characteristics}

Table 1 summarizes the characteristics of the study cohort. Adolescents averaged 16.8 (SD 0.26) y of age; $54 \%$ were male; and the mean $\mathrm{BMlz}$ was 0.52. Adipokines were significantly higher among females than males: leptin (female: 19.07, SD 14.58; male: 5.93, SD 9.24; $P<.001$ ), and L:A (female: 2.01, SD 2.32; male: 0.74, SD 1.17; $P<.001)$. There were also sex differences for the following metabolic risk factors: elevated BP (female: $4 \%$, male: $11 \%, P<.001$ ); low HDL (female: $75 \%$, male: $64 \%, P<.002$ ); elevated glucose (female $7 \%$, male: $1 \%, P<.03$ ); and abdominal obesity (female: $51 \%$; males: $13 \%$;
TABLE 1 Sample characteristics of the cohort (total sample and by sex; Santiago, Chile)

\begin{tabular}{|c|c|c|c|}
\hline & $\begin{array}{l}\text { Total sample } \\
N=679\end{array}$ & $\begin{array}{l}\text { Female } \\
n=322\end{array}$ & Male $n=357$ \\
\hline $\begin{array}{l}\text { Age at } 16 \mathrm{y} \\
\text { evaluation }\end{array}$ & $16.8(0.3)$ & $16.9(0.3)$ & $16.8(0.3)$ \\
\hline 10 y BMI z-score & $0.52(1.02)$ & $0.68(0.95)$ & $0.83(0.95)$ \\
\hline 16 y BMI z-score* & $0.76(0.95)$ & $0.61(0.94)$ & $0.45(1.08)$ \\
\hline Ghrelin & $238(146)$ & $251(154)$ & $227(137)$ \\
\hline Leptin*** & $12.17(13.74)$ & $19.07(14.58)$ & $5.93(9.24)$ \\
\hline Adiponectin & $11.29(5.36)$ & $10.28(4.95)$ & $12.39(5.57)$ \\
\hline $\mathrm{L}: \mathrm{A}^{* * *}$ & $1.35(1.91)$ & $2.01(2.32)$ & $0.74(1.17)$ \\
\hline HOMA-IR & $1.79(1.32)$ & $1.80(1.23)$ & $1.78(1.40)$ \\
\hline $\begin{array}{l}\text { Elevated } \\
\qquad \mathrm{BP}, \%, n^{* *}\end{array}$ & $7 \%, 50$ & $4 \%, 12$ & $11 \%, 38$ \\
\hline Low HDL, \%, $n^{* *}$ & $69 \%, 471$ & $75 \%, 242$ & $64 \%, 229$ \\
\hline Elevated TG, \%, $n$ & $8 \%, 53$ & $8 \%, 24$ & $8 \%, 29$ \\
\hline $\begin{array}{l}\text { Elevated } \\
\text { glucose, \%, n* }\end{array}$ & $9 \%, 61$ & $7 \%, 21$ & $11 \%, 40$ \\
\hline $\begin{array}{c}\text { Abdominal } \\
\text { obesity, } \\
\%, n^{* * *}\end{array}$ & $30 \%, 205$ & $51 \%, 158$ & $13 \%, 47$ \\
\hline $\begin{array}{l}\text { No. of metabolic } \\
\text { risk factors }\end{array}$ & $1.2(0.9)$ & $1.4(0.9)$ & $1.1(1.0)$ \\
\hline Maternal BMI & $29(5)$ & $29(5)$ & $29(5)$ \\
\hline
\end{tabular}

Note: Appetite hormones and energy regulators were examined by sex and using the 95th percentile cut-off for obesity; IDF criteria used to calculate cutoff values for each metabolic risk factor. Values represent mean (SD), unless indicated otherwise. *Values statistically different by sex at $P<.05, * * P<.01, * * * P<.001$.

Abbreviation: No., abbreviation for number.

$P<.001)$. BMlz-16y was correlated with BMlz-10y $(\rho=0.60$, $P<.001)$ and metabolic risk factors $(\rho=0.60, P<.001)$.

\subsection{2 | Main results}

Model fit for the path analysis for the total sample was acceptable (CFI = 0.99; RMSEA = 0.03; and SRMR = 0.03). Findings for males and females were similar; therefore, we present findings for the total sample illustrated in Figure 3. 
FIGURE 3 Pathways linking child weight status to risk for metabolic syndrome in a cohort of Chilean adolescents ( $n=679$ ); Model fit: $\mathrm{CFI}=0.97$, RMSEA $=0.05$, SRMR $=0.03, R$-square $=0.38$, total indirect effects $=0.17$. Note: Model controls for maternal BMI, sex, and age

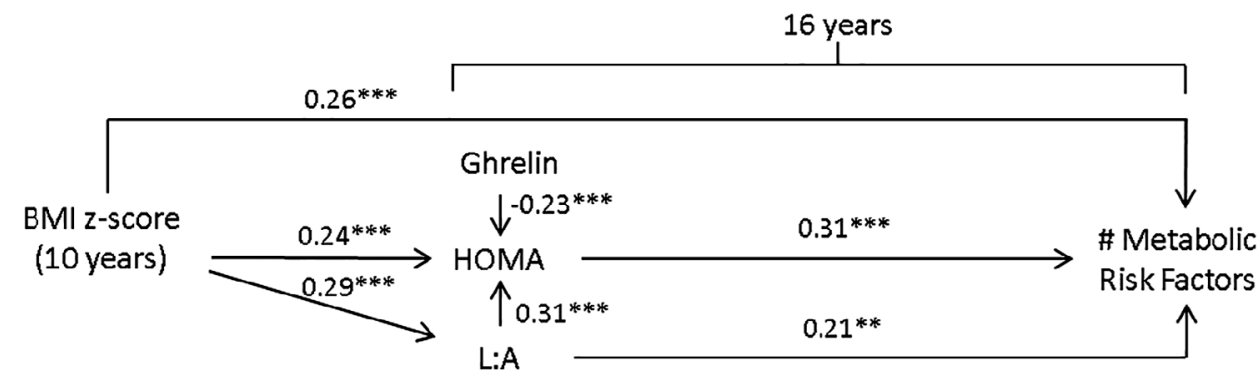

Higher BMI at 10 y was directly related to an increase in metabolic risk $(\beta=0.26, P<.001)$. In addition, BMlz-10y related to an increase in HOMA-IR $(\beta=0.24, P<.001)$ and $L: A(\beta=0.29, P<.001)$. In turn, an increase in HOMA-IR and an increase in L:A related to increased metabolic risk $(\beta=0.31, P<.001$ and $\beta=0.21, P<.001$, respectively). An increase in L:A was also associated with increased HOMA-IR ( $\beta=0.31, P<.001$ ). BMlz-10y was not related to ghrelin at $16 \mathrm{y}$, but an increase in ghrelin was related to a decrease in HOMA-IR $(\beta=-0.23, P<.001)$.

Female sex was significantly related to higher ghrelin levels $(\beta=0.09, P=.02)$, higher L:A $(\beta=0.35, P<.001)$, and greater metabolic risk $(\beta=0.13, P<.001)$. Age was negatively related to ghrelin levels $(\beta=-0.13, P=.001)$ and HOMA-IR $(\beta=0.08, P=.01)$, and maternal BMI was related to BMlz-10y $(\beta=0.25, P<.001)$. The final model explained $39 \%$ of the variance in metabolic risk.

\subsection{3 | Test of indirect effects}

The significant indirect effects included (all $P<.001$ ): BMlz-10y $\rightarrow$ L:A $\rightarrow$ metabolic risk $(\beta=0.06)$; $\mathrm{BMlz}-10 \mathrm{y} \rightarrow \mathrm{HOMA} \rightarrow$ metabolic risk $(\beta=0.07) ; \mathrm{BMlz}-10 \mathrm{y} \rightarrow \mathrm{L}: \mathrm{A} \rightarrow \mathrm{HOMA} \rightarrow$ metabolic risk $(\beta=0.03)$. Total indirect effects were 0.17 .

\section{4 | DISCUSSION}

This study examined potential metabolic pathways by which a higher childhood weight status might contribute to increased metabolic risk among adolescents. We found multiple metabolic pathways mediated by adipokines and insulin resistance. First, an increase in weight status at age 10 y directly related to increased number of risk factors for the metabolic syndrome at $16 \mathrm{y}$. Second, weight status was indirectly related to an increase in metabolic risk through both leptin/ adiponectin ratio and insulin resistance. Third, we found that insulin resistance partially mediated the relation between leptin/adiponectin ratio and ghrelin and metabolic risk. These findings show the deleterious effect of higher childhood weight status on adolescent metabolic health.

In this adolescent cohort, we found higher levels of leptin and ratios of leptin/adiponectin among females than males, but not of ghrelin. For risk factors comprising metabolic risk, we found that males more than females had elevated blood pressure and elevated glucose, yet females had lower HDL and greater abdominal obesity compared to males. In fact, half of females in our study were classified as having central obesity. When considering all factors, we found that females had higher metabolic risk. These findings are concerning and provides evidence of the disparities in cardiovascular and cardiometabolic health among females in this setting. Others have found similar findings. ${ }^{14,15}$ Furthermore, in light of the pathways illustrated by Lucasson et al. (see Figure 1), and controlling for sex, we observed that higher ratios of leptin/adiponectin worsened insulin resistance, which is in line with the functions of leptin (pro-inflammatory) and adiponectin (anti-inflammatory). ${ }^{8}$ Similarly, Schutte and colleagues found a positive relationship between leptin with insulin resistance in a study of 115 women. ${ }^{8}$ There are, however, other biological mechanisms beyond the scope of this study that may play a role in the pathway from child obesity to metabolic risk during adolescence. For instance, appetite hormone dysregulation could have contributed to increased caloric intake or appetite, and in turn increase risk for obesity, which increased risk for insulin resistance. Factors related to appetite hormone regulation most likely include lifestyle behaviors such as sleep, dietary patterns and physical activity.

We found that increased weight status in childhood predicted poor metabolic health in adolescence, which is consistent with the developmental origins of health and disease. Moreover, our findings elucidate mechanisms by which childhood obesity may be connected to appetite hormone regulation, insulin resistance, and metabolic risk. In a prior study in the same cohort, we also found evidence to support the negative impact of early onset obesity (measured at $5 \mathrm{y}$ ) on adolescent metabolic health. ${ }^{16}$ Increased weight status at age $10 \mathrm{y}$ predicted an increase in the leptin/adiponectin ratio at 16 years old, which we expected, given that levels of leptin (positively) and adiponectin (negatively) have been associated with increased body mass index in children. ${ }^{9}$ In turn, the leptin/adiponectin ratio was positively associated with an increased risk for poor metabolic health. In addition, children with higher weight status were prone to insulin resistance and, in turn, at risk for poor metabolic health, consistent with the hypothesis that insulin resistance is the underlying cause of metabolic syndrome. ${ }^{17}$ In this study, these pathways accounted for almost half of the variance in metabolic risk. Future research could include lifestyle behaviors known to be associated with metabolic risk, such as sleep, diet, and physical activity, for a more comprehensive understanding of the biological and behavioral mechanisms related to metabolic syndrome.

Another unique finding is that increased ghrelin concentration related to a decrease in insulin resistance, whereas leptin/adiponectin 
ratio was related to an increase in insulin resistance. These findings differ slightly from the framework developed by Lucasson et al. (Figure 1), as our findings suggest that ghrelin and leptin are connected to insulin resistance. In addition, Klok and colleagues have proposed that leptin and ghrelin systems function independently of each other in the control of energy homeostasis. ${ }^{3}$ Our findings support this notion, given that child weight status did not relate to ghrelin during adolescence but did relate to an increased leptin/adiponectin ratio. Furthermore, our findings are consistent with research showing high leptin in individuals with obesity, which suggests the phenomenon of leptin resistance. ${ }^{18}$

This study has some limitations to consider. The sample of Chilean adolescents was not representative of the Chilean adolescent population, as most were from low- to middle-income backgrounds in urban Santiago. Also, adolescents were recruited as infants to participate in an iron deficiency anemia trial. Inclusion criteria for study recruitment required that infants were term, healthy infants and weighed $\geq 3 \mathrm{~kg}$ at birth. Caution is needed when attempting to generalize to a larger population. Nevertheless, findings may be equally relevant given the high prevalences of obesity, type 2 diabetes, and cardiovascular disease among individuals from low middle socioeconomic status, as estimated by the Chilean National Health Survey. ${ }^{19}$ Another limitation is the cross-sectional nature of data on cardiometabolic biomarkers, which were not measured during prior assessments of the cohort. This limits the ability to draw conclusions related to the temporality of metabolic pathways. Future studies are needed to consider mechanisms over time.

\section{5 | CONCLUSIONS}

To our knowledge, this is one of the first studies to illustrate the connection of childhood obesity to metabolic pathways involving appetite hormone regulation and metabolic risk during adolescence. Increased weight status at 10 y directly and indirectly increased risk for metabolic syndrome among adolescents via metabolic pathways that included appetite hormones and insulin resistance. These findings not only demonstrate the harmful role of childhood obesity on adolescent risk for metabolic syndrome but also highlight the independent role of ghrelin as a protective factor for insulin resistance. Childhood interventions are needed to attenuate the harmful role of childhood overweight/obesity on adolescent metabolic health. Further research is needed to gain a better understanding of the independent role of ghrelin in metabolic risk as well as behavioral factors (ie, sleep, diet, physical activity) that play a role in inflammation and metabolic risk.

\section{ACKNOWLEDGEMENTS}

This research was funded by R01HL088530 (NIH-NHLBI, PI: Gahagan); R01HD33487 (NIH-NICHD, Pls: Lozoff and Gahagan); K01HL129087 (NHLBI, PI: Martinez). The first author acknowledges Marcela Reyes for her support. All authors would like to thank the study participants for their on-going commitment to the project.

\section{AUTHOR CONTRIBUTIONS}

Suzanna M. Martinez: Dr. Martinez conceptualized and designed the study, conducted the data analysis, contributed substantially to the interpretation of the data, drafted the initial manuscript, and approved the final manuscript as submitted.

Estela Blanco: Ms. Blanco substantially contributed to the acquisition of data, provided important feedback on data interpretation, and revised the article critically for important intellectual content. She has read and approved the final version.

Raquel Burrows: Dr. Burrows substantially contributed to the overall conception and design, and contributed to the acquisition of data. She also provided important feedback on data interpretation and revised the article critically for important intellectual content. She has read and approved of the final version.

Sheila Gahagan: Dr. Gahagan substantially contributed to the conception and design of the project, acquisition of data and assisted with data analysis and interpretation. Gahagan revised the article critically for important intellectual content on several occasions. She has read and approves of the final version.

\section{ORCID}

Suzanna M. Martinez (D) https://orcid.org/0000-0001-7864-1391

\section{REFERENCES}

1. Barker DJ. The origins of the developmental origins theory. J Intern Med. 2007;261(5):412-417.

2. Roberts CK, Hevener AL, Barnard RJ. Metabolic syndrome and insulin resistance: underlying causes and modification by exercise training. Compr Physiol. 2013;3(1):1-58.

3. Klok MD, Jakobsdottir S, Drent ML. The role of leptin and ghrelin in the regulation of food intake and body weight in humans: a review. Obesity Rev. 2007;8(1):21-34.

4. Dixit VD, Schaffer EM, Pyle RS, et al. Ghrelin inhibits leptin- and activation-induced proinflammatory cytokine expression by human monocytes and T cells. J Clin Invest. 2004;114(1):57-66.

5. Schwartz MW, Peskind E, Raskind M, Boyko EJ, Porte D Jr. Cerebrospinal fluid leptin levels: relationship to plasma levels and to adiposity in humans. Nat Med. 1996;2(5):589-593.

6. Meyer LK, Ciaraldi TP, Henry RR, Wittgrove AC, Phillips SA. Adipose tissue depot and cell size dependency of adiponectin synthesis and secretion in human obesity. Adipocyte. 2013;2(4):217-226.

7. Lucassen EA, Rother KI, Cizza G. Interacting epidemics? Sleep curtailment, insulin resistance, and obesity. Ann N Y Acad Sci. 2012;1264(1): 110-134.

8. Schutte AE, Huisman HW, Schutte R, et al. Adipokines and cardiometabolic function: how are they interlinked? Regul Pept. 2010; 164(2):133-138.

9. Schoppen S, Riestra P, Garcia-Anguita A, et al. Leptin and adiponectin levels in pubertal children: relationship with anthropometric variables and body composition. Clin Chem Lab Med. 2010;48(5):707-711.

10. Larsen MA, Isaksen VT, Moen OS, et al. Leptin to adiponectin ratio: a surrogate biomarker for early detection of metabolic disturbances in obesity. Nutr Metabolism Cardiovasc Dis. 2018;28(11):1114-1121.

11. Lozoff B, De Andraca I, Castillo M, Smith JB, Walter T, Pino P. Behavioral and developmental effects of preventing iron-deficiency anemia in healthy full-term infants. Pediatrics. 2003;112(4):846-854.

12. Burrows $R$, Correa-Burrows $P$, Reyes $M$, Blanco $E$, Albala $C$, Gahagan S. Low muscle mass is associated with cardiometabolic risk 
regardless of nutritional status in adolescents: a cross-sectional study in a Chilean birth cohort. Pediatr Diabetes. 2017;18(8):895-902.

13. Fernández JR, Redden DT, Pietrobelli A, Allison DB. Waist circumference percentiles in nationally representative samples of AfricanAmerican, European-American, and Mexican-American children and adolescents. J Pediatr. 2004;145(4):439-444.

14. Song X, Tabák AG, Zethelius B, et al. Obesity attenuates gender differences in cardiovascular mortality. Cardiovasc Diabetol. 2014;13(1):144.

15. Schorr M, Dichtel LE, Gerweck AV, et al. Sex differences in body composition and association with cardiometabolic risk. Biol Sex Differ. 2018;9(1):28.

16. Pacheco LS, Blanco E, Burrows R, Reyes M, Lozoff B, Gahagan S. Early onset obesity and risk of metabolic syndrome among Chilean adolescents. Prev Chronic Dis. 2017;14:E93.

17. Reaven GM. Banting lecture 1988. Role of insulin resistance in human disease. Diabetes. 1988;37(12):1595-1607.
18. Lee JH, Reed DR, Price RA. Leptin resistance is associated with extreme obesity and aggregates in families. Int $J$ Obes Relat Metab Disord. 2001;25(10):1471-1473.

19. Ministry of Health (Chile). National Health Survey 2009-2010. http://ghdx.healthdata.org/record/chile-national-health-survey2009-2010. Accessed September 21, 2018.

How to cite this article: Martinez SM, Blanco E, Burrows R, Lozoff B, Gahagan S. Mechanisms linking childhood weight status to metabolic risk in adolescence. Pediatr Diabetes. 2020; 21:203-209. https://doi.org/10.1111/pedi.12972 